\title{
Phased matched UV generation in a silica holey fiber
}

\author{
J.H.V. Price, K. Furusawa, T.M. Monro, C. Netti, A. Malinowski, J.J. Baumberg, D.J. Richardson \\ Optoelectronics Research Centre, University of Southampton, UK
}

\begin{abstract}
We demonstrate both simultaneous supercontinuum generation and efficient phase-matched UV generation around $350 \mathrm{~nm}$ in a highly nonlinear silica holey fiber. The resulting spectrum extends from $<300 \mathrm{~nm}$ out to $>$ $1800 \mathrm{~nm}$ and, as far as we are aware, is the broadest optical spectrum yet reported for a fiber based supercontinuum generator.
\end{abstract}

The generation of ultra broadband supercontinuum light from microstructured fibers and tapered standard fibers offers many new opportunities for significant new developments in areas such as spectroscopy [1] and metrology [2]. To date, spectra have been reported spanning from 390-1600mn using microstructured fiber [3], and from 375$1500 \mathrm{~nm}$ [4] using tapered fiber, however there remains strong interest in extending the achievable bandwidths still further, particularly towards the ultra-violet (UV) regions of the spectrum. Here using UV transmitting pure silica holey fiber we report strong UV generation at a peak wavelength of $350 \mathrm{~nm}$, accompanied by supercontinuum generation out to at least $1800 \mathrm{~nm}$. We believe that this is the first time that the generation of $U V$ wavelengths below $300 \mathrm{~nm}$ has been demonstrated from a fiber device.

The robust, jacketed, polarization-maintaining holey fiber used in this demonstration has a small $\sim 1.6 \mu \mathrm{m}$ diameter core, and a large air fill fraction in the cladding, which combine to produce a highly confined mode with $\mathrm{A}_{\text {eff }} \sim 3 \mu \mathrm{m}^{2}$. The fiber has a predicted dispersion zero of $\sim 800 \mathrm{~nm}$ and a loss of $\sim 40 \mathrm{~dB} / \mathrm{km}$ at a wavelength of $1.55 \mu \mathrm{m}$. The fiber is rigorously single mode at wavelengths above $1 \mu \mathrm{m}$, but will support higher order modes at shorter wavelengths.
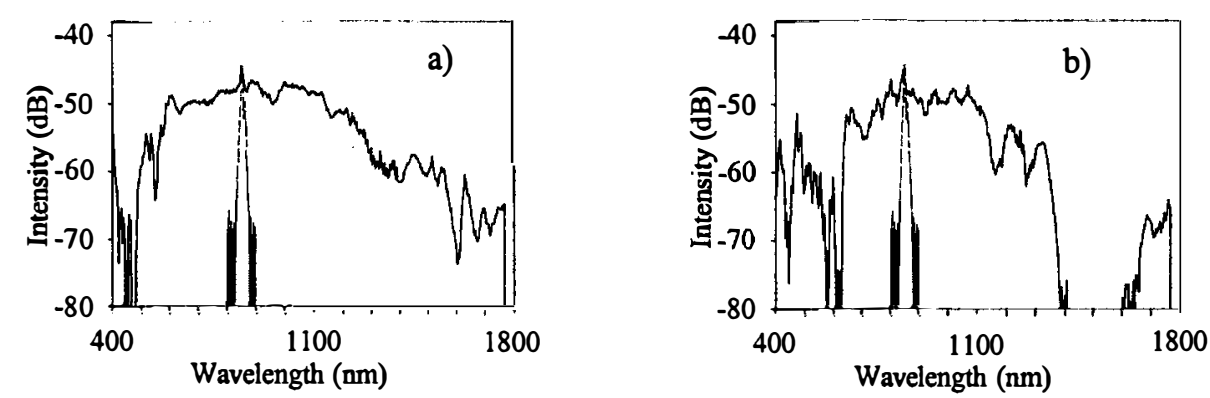

Fig. 1. Supercontinuum spectrum from microstructured fiber. Dashed line is seed pulse. a) Maximum launch efficiency produces broad spectrum in fundamental mode. b) Off-axis launch produces enhanced blue generation.

We injected high energy, $120 \mathrm{fs}$ pulses at $850 \mathrm{~nm}$ generated by an amplified Ti:Sapphire laser operating at 250 $\mathrm{KHz}$ repetition rate into $20 \mathrm{~cm}$ of our holey fiber, and measured the resulting optical spectrum. Initially we used a fiber coupled optical spectrum analyzer with a measurement range from 400-1800nm. Fig. 1.a) shows the supercontinuum produced when we launched the maximum pulse energy of $10 \mathrm{~nJ}$. The spectrum is flat from $600-$ $1200 \mathrm{~nm}$, and extends from $\sim 480 \mathrm{~nm}$ to at least $1800 \mathrm{~nm}$. The output appeared yellow coloured and was spatially single mode in the far-field. However, by slightly detuning the pump launch conditions from that for optimal launched power we were able to obtain a visually stunning change in the colour of the source output from yellow to bright white. The corresponding optical spectrum is shown in Fig.1.b) where it is seen that the width of the flat broadband supercontinuum is reduced (due to the reduced peak power), but that the spectrum is significantly enhanced in the blue region of the spectrum. Furthermore, using colored filters we found that the red pump light was in the fundamental mode, but that the new spectral components were in a $2^{\text {nd }}$ order spatial mode. 

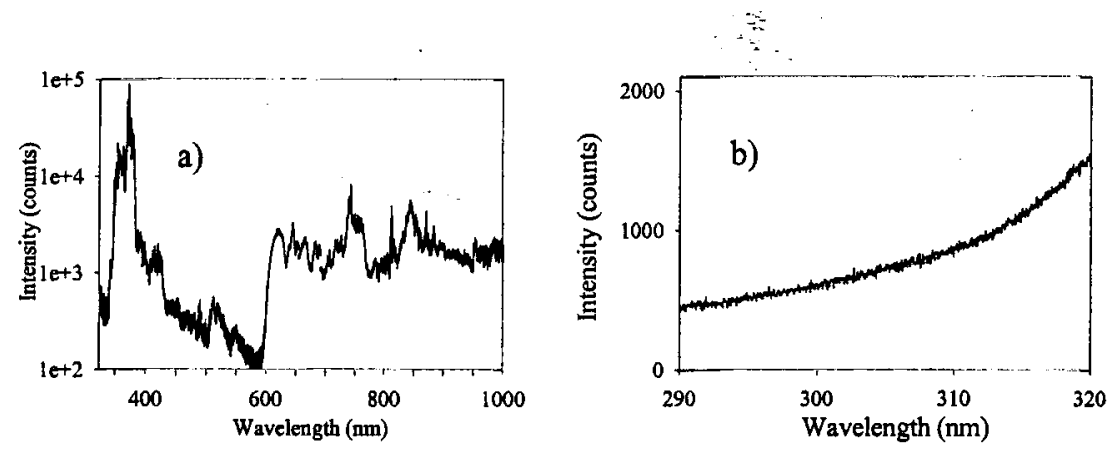

Fig. 2. UV spectra. a) Broad spectrum after correcting for instrument response and dark counts. b) Narrow spectrum, without any adjustment, showing UV generation below $300 \mathrm{~nm}$.

To further analyze the spectral content of the white light spectrum (since it was visibly clear that significant UV was present in the beam) we used a free space coupled grating monochromator with a CCD detector with associated achromatic/UV compatible optics. The resulting spectrum is shown in Fig. 2.a) where an intense UV peak at $356 \mathrm{~nm}$ is observed with a long tail extending out to well below $300 \mathrm{~nm}$ (see Fig.2.b). This peak was not present when the seed was optimally coupled into the holey fiber and was always accompanied by the production of blue light in the second order spatial mode. The high efficiency, and the involvement of other wavelengths and modes in the phenomena suggest some form of phase-matched intermodal process, although we have yet to identify the precise details.

In conclusion, we have demonstrated, UV generation and associated supecontinuum generation from a holey fiber at wavelengths down to below $300 \mathrm{~nm}$. This substantially increases the reported spectral range simultaneously generated in a single fiber.

1. R. Holzwarth, T. Udem, T. W. Hansch, J. C. Knight, W. J. Wadsworth and P. S. J. Russell, "Optical frequency synthesizer for precision spectroscopy," Physical Review Letters 85, 2264-2267 (2000).

$2 . \quad$ D. J. Jones, S. A. Diddams, J. K. Ranka, A. Stentz, R. S. Windeler, J. L. Hall and S. T. Cundiff, "Carrier-envelope phase control of femtosecond mode-locked lasers and direct optical frequency synthesis," Science 288, 635-639 (2000).

3. J. K. Ranka, R. S. Windeler and A. J. Stentz, "Visible continuum generation in air-silica microstructure optical fibers with anomalous dispersion at $800 \mathrm{~nm}$," Optics Letters 25, 25-27 (2000).

4. T. A. Birks, W. J. Wadsworth and P. S. Russell, "Supercontinuum generation in tapered fibers," Optics Letters 25, 1415-1417 (2000). 